\title{
Efectos de la suplementación de treonina sobre el rendimiento productivo, carcasa y pesos de órganos de cuyes de engorde con alimentación mixta
}

\author{
Effects of threonine supplementation on productive performance, carcass and \\ organ weights of fattening guinea pigs with mixed feeding
}

\author{
Manuel Paredes ${ }^{1,2}$, María Cerquín ${ }^{1}$
}

\section{Resumen}

\begin{abstract}
El experimento tuvo como objetivo evaluar los efectos de cinco niveles dietéticos de treonina (Thr) sobre el rendimiento en engorde, características de la carcasa y el desarrollo del tracto gastrointestinal (TGI) y visceral de cuyes. Se trabajó con 40 cuyes machos mejorados de 30 días de edad distribuidos al azar en 5 tratamientos con cuatro repeticiones en un diseño completamente aleatorio y sacrificados a los 72 días de edad. La dieta consistió en pienso restringido y forraje verde ad libitum. La suplementación de Thr se realizó con L-Treonine $98.5 \%$ (L-Thr) en niveles de $0,2,4,6$ y 8 g/ $\mathrm{kg}$ de pienso, para obtener niveles de $0.55,0.59,0.63,0.67$ y $0.71 \%$ de Thr. Los cuyes con $0.63 \%$ de Thr dietético lograron mayores ganancias de peso, mejor conversión alimenticia y mayor rendimiento de carcasa. El TGI con posibles funciones inmunológicas alcanzó un mayor peso relativo con niveles de 0.67 y $0.71 \%$ de Thr. El peso relativo del bazo y del corazón no se afectaron por los niveles de Thr, mientras que se observaron diferencias significativas en hígado, pulmones y riñones.
\end{abstract}

Palabras clave: cuy, treonina, alimentación mixta, engorde, carcasa

\footnotetext{
${ }^{1}$ Facultad de Ingeniería en Ciencias Pecuarias, Universidad Nacional de Cajamarca, Perú

${ }^{2}$ E-mail: mparedes@unc.edu.pe
}

Recibido: 19 de mayo de 2021

Aceptado para publicación: 13 de noviembre de 2021

Publicado: 22 de diciembre de 2021

CLos autores. Este artículo es publicado por la Rev Inv Vet Perú de la Facultad de Medicina Veterinaria, Universidad Nacional Mayor de San Marcos. Este es un artículo de acceso abierto, distribuido bajo los términos de la licencia Creative Commons Atribución 4.0 Internacional (CC BY 4.0) [https:// creativecommons.org/licenses/by/4.0/deed.es] que permite el uso, distribución y reproducción en cualquier medio, siempre que la obra original sea debidamente citada de su fuente original 
The experiment aimed to evaluate the effects of five dietary levels of threonine (Thr) on growth performance, carcass characteristics, and gastrointestinal (GIT) and visceral tract development of fattening guinea pigs. In total, 40 improved 30-day-old male guinea pigs were randomly distributed in five treatments with four repetitions in a completely random design and slaughtered at 72 days of age. The diet consisted of restricted concentrated feed and green forage ad libitum. Thr supplementation was performed with L-Treonine $98.5 \%$ (L-Thr) at levels of $0,2,4,6$ and $8 \mathrm{~g} / \mathrm{kg}$ of feed, to obtain levels of 0.55 , $0.59,0.63,0.67$ and $0.71 \%$ of Thr. Guinea pigs with $0.63 \%$ dietary Thr achieved greater weight gains, better feed conversion and higher carcass performance. The GIT with possible immunological functions reached a higher relative weight with levels of 0.67 and $0.71 \%$ of Thr. The relative weight of the spleen and heart were not affected by Thr levels, while significant differences were observed in liver, lungs and kidneys.

Key words: guinea pig, threonine, mixed feeding, fattening, carcass

\section{INTRODUCCIÓN}

En el Perú se ha incrementado la producción de carne de cuy, con más del 70\% de criadores en las provincias de San Marcos y Cajabamba, que utilizan el sistema de producción familiar-comercial (Ortiz et al., 2021), aprovechando las características biológicas de esta especie, que le permiten nutrirse con pasturas, pasturas suplementadas con pienso o únicamente con pienso (Chauca, 2018).

Los piensos o alimentos integrales por lo general tienen un bajo contenido de fibra. La fibra está presente de forma natural en los alimentos de origen vegetal y es un componente importante en la dieta de animales monocavitarios herbívoros (Saadatmand et al., 2019). Dependiendo de la cantidad, la fibra dietética puede mejorar el funcionamiento de los órganos digestivos y el rendimiento del animal en crecimiento (Kheravii et al., 2017). El cuy por ser una especie herbívora puede consumir grandes cantidades de fibra, la cual es fermentada por la microbiota cecal y puede proteger el tracto gastrointestinal (TGI) de la colonización de patógenos
(Liévin-Le Moal y Servin, 2006). La pectina de las pasturas es una fracción de fibra soluble que también forma la digesta dentro del TGI (Serena et al., 2007). Del mismo modo, las pasturas contienen fibras insolubles, conocidas principalmente por sus efectos sobre el desarrollo intestinal y efectos abrasivos sobre la mucosa intestinal (Montagne et al., 2003).

La mucina está compuesta por proteínas y algunos aminoácidos como serina, cisteína y treonina (Thr), constituyendo la $\mathrm{Thr}$ el $11 \%$ de los aminoácidos de la mucina, variando la cantidad y tipo de mucina de acuerdo con la disponibilidad de Thr y fibra en el alimento (Saadatmand et al., 2019), de allí la importancia de la Thr en el mantenimiento de la integridad intestinal (Wang et al., 2010). La síntesis de proteínas corporales, colágeno, elastina y anticuerpos, así como la producción de ácido úrico y enzimas pancreáticas también requieren Thr (Debnath et al., 2019).

La influencia directa de Thr en los parámetros de crecimiento y rasgos de la carcasa se ha determinado en aves (Ji et al., 2019). La Thr es fundamental para la utilización óptima de aminoácidos que contienen 
azufre (Met + Cis) y lisina (Kidd, 2000). De la Thr depende el buen funcionamiento del intestino y el tejido linfoide asociado al intestino como componentes esenciales del sistema inmunológico, que cumplen la función de normalizar la secreción de citocinas inflamatorias y mejorar el número de linfocitos $\mathrm{T}$, las funciones específicas de las células T y la secreción de IgA (Ruth y Field, 2013). Por otro lado, la Thr es el tercer aminoácido limitante después de la metionina y lisina en pollos de engorde (Berres et al., 2007), particularmente en dietas bajas en proteína cruda (Rezaeipour et al., 2012).

Se ha comprobado que la $\mathrm{Thr}$ regula los niveles de expresión de genes relacionados con el transporte de aminoácidos y la deposición de proteínas; siendo la concentración óptima de Thr en la dieta de gallinas reproductoras de $0.68 \%$ (Jiang et al., 2019). También se ha determinado que la adición de prebióticos de pared celular en la dieta de pollos de engorde hace variar el requerimiento de aminoácidos; así, una relación Thr a lisina de 0.7 mejoró el rendimiento productivo con un aumento de ancho de vellosidad en yeyuno y altura de vellosidad en íleon (Abdaljaleel $e t$ al., 2018). La Thr dietética, por encima de los requerimientos, promueve el crecimiento de los órganos inmunes, estimula la síntesis de inmunoglobulinas, mejora la respuesta inmunitaria y alivia el estrés inmunológico causado por Escherichia coli (Trevisi et al., 2015). En cuyes de laboratorio se ha determinado que $0.5 \%$ de Thr dietaria es el nivel óptimo en fase de crecimiento (NRC, 1995).

Los niveles de Thr dietaria según lo informado por la NRC (1995) podrían ser insuficientes para cuyes criados con fines cárnicos, toda vez que dicha información se aplica mayormente a cuyes de laboratorio. Asimismo, es importante determinar si la Thr tiene un papel directo en la mejora del engorde y carcasa, y desarrollo de órganos digestivos y del sistema inmunitario del cuy como especie productora de carne. De esta manera, la presente investigación tuvo como objetivo conocer los efectos de cinco niveles de
Thr en el pienso como suplemento a una dieta basada en pasturas sobre indicadores de engorde, características de carcasa, desarrollo del TGI y órganos internos con posibles funciones inmunitarias de cuyes criados con fines cárnicos.

\section{Materiales Y MéTodos}

\section{Cuyes, Diseño Experimental y Dietas}

El estudio se llevó a cabo en las instalaciones de una granja de cuyes de propiedad privada ubicada en el valle de Cajamarca, Perú, a $2772 \mathrm{msnm}$. Se emplearon 40 cuyes machos de la raza Perú, de $30 \pm 3$ días de edad y $526 \mathrm{~g}$ de peso inicial promedio. Los cuyes fueron distribuidos al azar en cinco tratamientos, cada uno con cuatro repeticiones y cada repetición con dos animales. Los cuyes fueron alojados en pozas de ladrillo divididas con malla galvanizada, cada poza con dos divisiones; en cada división se alojó dos cuyes (una unidad experimental), cada división con comedero y bebedero de tierra cocida. La duración del experimento fue de seis semanas (30 a 72 días de edad).

Los cuyes fueron mantenidos bajo el sistema de alimentación mixta, con el suministro diario y restringido de pienso tipo harina a las 08:00 y la oferta ad libitum de forraje verde de rye grass (Lolium multiflorum) y alfalfa (Medicago sativa). La mezcla de forraje verde de ambas pasturas y alfalfa (FVRGA) se suministró a las 09:00 y 16:00 h. El agua de bebida estuvo a disposición de los cuyes durante todo el día. El pienso fue preparado en una fábrica de alimentos balanceados en Pacasmayo, La Libertad (Cuadro 1).

La suplementación con Thr se hizo con L-Threonine (L-Thr), grado alimenticio 98.5\% (Ajinomoto Heartland, USA). Se suplementó el pienso con cinco niveles de L-Thr: 0 (pienso basal), 2, 4, 6 y $8 \mathrm{~g} / \mathrm{kg}$ de pienso, lo cual dio lugar a cinco concentraciones de $\mathrm{Thr}$ en el pienso: $0.64,0.84,1.04,1.24$ y $1.44 \%$. 
Cuadro 1. Ingredientes y contenido nutricional del pienso basal(base fresca) utilizado en el estudio

\begin{tabular}{lc}
\hline & $\begin{array}{c}\text { Cantidad } \\
(\mathrm{g} / 100 \mathrm{~g})\end{array}$ \\
\hline Ingredientes & 16.0 \\
Maíz amarillo & 16.0 \\
Torta de soya & 30.0 \\
Polvillo de arroz & 35.0 \\
Afrecho de trigo & 2.3 \\
Carbonato de calcio & 0.1 \\
DL Metionina & 0.5 \\
Sal común, NaCl & 0.1 \\
Premezcla vitaminas y & \\
minerales ${ }^{1}$ & \\
Contenido nutricional calculado & \\
Materia seca & 88.44 \\
Proteína cruda & 17.26 \\
Energía digestible, kcal/kg & 2823 \\
Fibra cruda & 7.51 \\
Metionina & 0.38 \\
Lisina & 0.86 \\
Treonina & 0.64 \\
Ca & 1.00 \\
P & 0.89 \\
Na & 0.20 \\
\hline
\end{tabular}

${ }^{1}$ Cada kilogramo contiene: Vit. A 9000 mil UI, Vit. $\mathrm{D}_{3} 2500$ mil UI, Vit. E 15000 UI, Vit. K 2.5 g, tiamina $1.5 \mathrm{~g}$, riboflavina $6.5 \mathrm{~g}$, cianocobalamina $0.01 \mathrm{~g}$, ácido pantoténico $5.50 \mathrm{~g}$, ácido fólico $1 \mathrm{~g}$, niacina 25 g, Mn 70 g, Zn 70 g, Fe 30 g, Cu 8 g, I 1 $\mathrm{g}$, Se $0.30 \mathrm{~g}$, Co $0.1 \mathrm{~g}$

Las cantidades de alimento por tratamiento se determinaron de acuerdo con el suministro diario de alimento:

- $\quad$ T1, 20 g de pienso basal + FV-RGA

- $\quad$ T2, $20 \mathrm{~g}$ de pienso basal $+2 \mathrm{~g} / \mathrm{kg}$ de L$\mathrm{Thr}+\mathrm{FV}-\mathrm{RGA}$

- $\quad$ T3, $20 \mathrm{~g}$ de pienso basal $+4 \mathrm{~g} / \mathrm{kg}$ de L$\mathrm{Thr}+\mathrm{FV}-\mathrm{RGA}$
- $\quad$ T4, $20 \mathrm{~g}$ de pienso basal $+6 \mathrm{~g} / \mathrm{kg}$ de L$\mathrm{Thr}+\mathrm{FV}-\mathrm{RGA}$

- $\quad$ T5, $20 \mathrm{~g}$ de pienso basal $+8 \mathrm{~g} / \mathrm{kg}$ de LThr + FV-RG

ElFV-RGA fue obtenido de los potreros de la misma granja. Semanalmente se evalúo la composición florística del forraje, habiéndose determinado en promedio durante todo el experimento una mezcla forrajera con $82 \%$ de rye grass y $18 \%$ de alfalfa. El contenido nutricional del pienso fue calculado de acuerdo con la composición nutricional de los ingredientes indicada por de Blas (2010). El análisis de materia seca (MS) y proteína cruda (PC) del forraje fue realizado cada siete días en el Laboratorio de Control de Alimentos de la Universidad Nacional de Cajamarca. El valor de la Thr de los forrajes fue determinado en base a lo indicado por NRC (2001): Thr del rye grass $=3.8 \times \mathrm{PC} / 100 \mathrm{y}$ Thr de alfalfa $=4.3 \times \mathrm{PC} / 100$. El valor de la MS, PC $\mathrm{y}$ Thr de rye grass, alfalfa y mezcla forrajera se muestran en el Cuadro 2.

\section{Ingesta, Pesos Corporal y Conversión Alimenticia}

La ingesta de alimento fue controlada en forma diaria y el peso vivo semanalmente, utilizando una balanza electrónica Kern (capacidad $2100 \mathrm{~g} \pm 0.01 \mathrm{~g}$ ).

- El consumo voluntario FV-RGA se determinó restando la cantidad de alimento ofrecido menos la cantidad no consumida.

- El consumo de MS se calculó a partir de la suma de las cantidades ingeridas de pienso y FV-RGA multiplicadas por el contenido de MS de cada alimento.

- La ingesta diaria de alimento (IDA) por cuy y tratamiento se calculó con base al consumo promedio las seis semanas del estudio y dividido por 42 días.

- Conociendo la cantidad de MS consumida de cada alimento se determinó la proporción de FV-RGA a pienso.

- El consumo de Thr se calculó de acuerdo con los consumos de pienso y de FVRGA multiplicados por su contenido de 
Cuadro 2. Valores ${ }^{1}$ de materia seca, proteína cruda y treonina (en base seca) de rye grass, alfalfa y mezcla forrajera $82 \%$ rye grass y $18 \%$ alfalfa (FV-RGA)

\begin{tabular}{lccc}
\hline Forrajes & $\begin{array}{c}\text { Materia seca } \\
(\%)\end{array}$ & $\begin{array}{c}\text { Proteína cruda } \\
(\%)\end{array}$ & $\begin{array}{c}\text { Treonina } \\
(\%)\end{array}$ \\
\hline Rye grass & 19.81 & 11.82 & 0.45 \\
Alfalfa & 21.73 & 19.51 & 0.84 \\
Mezcla FV-RGA & 20.16 & 13.20 & 0.52 \\
\hline
\end{tabular}

${ }^{1}$ Los valores de materia seca y proteína cruda fueron obtenidos en el laboratorio de control de alimentos de la Universidad Nacional de Cajamarca. Los valores de treonina fueron calculados según NRC (2001): treonina del rye grass $=3.8 \times$ proteína cruda $/ 100$ y treonina de alfalfa $=4.3 \times$ proteína $\mathrm{cruda} / 100$

Thr, el cual fue expresado en porcentaje de acuerdo al consumo total de alimento en cada tratamiento $(\%$ de Thr de la dieta).

- La ganancia de peso media diaria (GMD) se calculó con base al peso inicial y final.

- $\quad$ El índice de conversión alimenticia (ICA) se determinó mediante IDA/GMD.

\section{Rendimiento de Carcasa y Peso de Ór- ganos}

Los animales fueron beneficiados al final del periodo experimental previo ayuno de 12 horas (cuatro cuyes por tratamiento). La carcasa incluyó piel, patas, cabeza, miembros anteriores y posteriores, excluyéndose las vísceras rojas (corazón, pulmones, hígado, riñones y bazo). El rendimiento de carcasa (RC) fue calculado con relación al peso vivo medido al momento de sacrificio (PVS): $\mathrm{RC}=$ (peso de carcasa / PVS) x 100. Se pesaron los órganos internos de cada cuy (corazón, pulmones, hígado, riñones y tracto gastrointestinal (TGI) comprendido desde la unión del esófago y estómago hasta la parte final del intestino grueso. Cada peso tomado de las partes del cuy fue expresado en porcentaje del PVS. Para el pesaje de los cuyes y órganos se utilizó la balanza Kern (capacidad $2100 \mathrm{~g}, \pm 0.01 \mathrm{~g}$ ).

\section{Análisis Estadistico}

El análisis estadístico de los datos se realizó bajo un diseño completamente al azar, con cinco tratamientos y cuatro repeticiones por tratamiento. El análisis de varianza se llevó a cabo usando el procedimiento GLM del Statistical Analysis System (SAS, 2003) y la comparación de medias se realizó utilizando la prueba de rango múltiple de Duncan.

\section{Resultados y Discusión}

\section{Ingesta, Pesos Corporal y Conversión Alimenticia}

La ingesta promedio de alimento tal como ofrecido y en base seca por cuy se indica en el Cuadro 3. Los cuyes consumieron los $20 \mathrm{~g}$ de alimento suministrado diariamente en todas las unidades experimentales. No se observaron diferencias estadísticas entre tratamientos en el consumo de FV-RGA ni en la ingesta de la suma de ambos alimentos $(\mathrm{p}>0.05)$.

La Thr consumida proveniente del FVRGA, pienso y de la suma de ambos alimentos, así como el porcentaje de Thr de la dieta se presenta en el Cuadro 3. Hubo diferencias entre tratamientos en la ingesta de Thr 
Cuadro 3. Ingestas de alimento tal como ofrecido (TCO) de materia seca (MS) y de treonina en cuyes de 30 a 72 días de edad ${ }^{1}$ alimentados con dieta mixta ${ }^{2} 80 \%$ rye grassalfalfa y $20 \%$ pienso

\begin{tabular}{|c|c|c|c|c|c|c|c|}
\hline & \multicolumn{5}{|c|}{ Nivel de treonina en el pienso (\%) } & \multirow{2}{*}{ SEM } & \multirow{2}{*}{$\mathrm{p}$} \\
\hline & 0.64 & 0.84 & 1.04 & 1.24 & 1.44 & & \\
\hline \multicolumn{8}{|l|}{ Ingesta en TCO, g/cuy } \\
\hline Alfalfa-rye grass & 12640.8 & 12380.6 & 12463.1 & 12803.8 & 12921.8 & 101.2 & 0.803 \\
\hline Pienso & 800.0 & 800.0 & 800.0 & 800.0 & 800.0 & -- & -- \\
\hline \multicolumn{8}{|l|}{ Ingesta de MS, g/cuy } \\
\hline Alfalfa-rye grass & 2717.8 & 2661.8 & 2679.6 & 2752.8 & 2778.2 & 21.8 & 0.803 \\
\hline Pienso & 707.2 & 707.2 & 707.2 & 707.2 & 707.2 & -- & -- \\
\hline Total & 3424.9 & 3369.0 & 3386.8 & 3460.0 & 3485.4 & 21.8 & 0.803 \\
\hline \multicolumn{8}{|c|}{ Ingesta de treonina, $\mathrm{g} /$ cuy } \\
\hline Alfalfa-rye grass & 14.1 & 13.8 & 13.9 & 14.3 & 14.4 & 0.11 & 0.916 \\
\hline Pienso & $4.5^{\mathrm{e}}$ & $5.9^{\mathrm{d}}$ & $7.3^{\mathrm{c}}$ & $8.7^{\mathrm{b}}$ & $10.2^{\mathrm{a}}$ & 1.00 & 0.003 \\
\hline Total & $18.7^{\mathrm{e}}$ & $19.8^{\mathrm{d}}$ & $21.3^{\mathrm{c}}$ & $23.1^{\mathrm{b}}$ & $24.6^{\mathrm{a}}$ & 1.08 & 0.014 \\
\hline $\begin{array}{l}\text { Treonina en la dieta } \\
(\%)\end{array}$ & $0.55^{\mathrm{e}}$ & $0.59^{\mathrm{d}}$ & $0.63^{\mathrm{c}}$ & $0.67^{\mathrm{b}}$ & $0.71^{\mathrm{a}}$ & 0.03 & 0.015 \\
\hline
\end{tabular}

${ }^{1}$ Cada valor representa la media de cuatro repeticiones y cada repetición estuvo conformada por dos cuyes machos

${ }^{2}$ La dieta mixta consistió en la oferta restringida de pienso (igual cantidad por repetición) + forraje ad libitum

SEM: Error estándar de la media

$a, b, c, d, e$ Las medias dentro de una fila que no comparten igual superíndice difieren significativamente $(p<0.05)$

proveniente del pienso, de la cantidad total y del porcentaje de Thr dietaria $(\mathrm{p}<0.05)$.

La ingesta de alimento no se vio afectada por los niveles de Thr en la dieta debido posiblemente a que la composición de ingredientes, contenido nutricional y formas de suministro fueron similares en los grupos experimentales, evitando variaciones alimenticias y uso de diferentes fuentes proteicas que pudieran influenciar sobre la ganancia de peso y el aprovechamiento de nutrientes (Ahmed et al., 2020). Estas condiciones son importantes para determinar con eficiencia el efecto de la Thr dietética sobre los demás indicadores de engorde. La alimentación mixta con pienso restringido y suministro de forraje a discreción permitió encontrar ingestas de forraje y pienso en proporciones de 80 a $20 \%$ con variaciones inferiores a $1 \%$ entre tratamientos. Los piensos concentrados con diferentes niveles de Thr no modificaron el comportamiento de ingesta del cuy, lo que permitió evaluar cuyes alimentados con Thr dietaria en niveles por encima de $0.5 \%$, que es el nivel recomendado para cuyes de laboratorio (NRC, 1995), así como con niveles mayores a $0.6 \%$ de Thr, que es la cantidad considerada en el pienso utilizado para sistemas mixtos e integrales en alimentación de cuyes con propósitos cárnicos (Huamaní et al., 2016). 
Cuadro 4. Promedios de peso final, ganancia de peso media diaria (GMD), ingesta diaria de alimento (IDA) e índice de conversión alimenticia (ICA) en cuyes de 30 a 72 días de edad ${ }^{1}$ alimentados con dieta mixta ${ }^{2} 80 \%$ rye grass-alfalfa y $20 \%$ pienso

\begin{tabular}{|c|c|c|c|c|}
\hline $\begin{array}{c}\text { Porcentaje de treonina } \\
\text { en la dieta }\end{array}$ & $\begin{array}{l}\text { Peso final } \\
(\mathrm{g})\end{array}$ & $\begin{array}{c}\text { GMD } \\
(\mathrm{g})\end{array}$ & $\begin{array}{c}\text { IDA } \\
(\mathrm{g} \text { MS) }\end{array}$ & ICA \\
\hline 0.55 & $1007.50^{c}$ & $11.49^{\mathrm{d}}$ & 81.55 & $7.09^{\mathrm{a}}$ \\
\hline 0.59 & $1051.25^{\mathrm{b}}$ & $12.35^{\mathrm{c}}$ & 79.37 & $6.42^{\mathrm{b}}$ \\
\hline 0.63 & $1168.75^{\mathrm{a}}$ & $15.21^{\mathrm{a}}$ & 79.80 & $5.24^{\mathrm{c}}$ \\
\hline 0.67 & $1081.25^{\mathrm{a}}$ & $13.18^{\mathrm{b}}$ & 81.54 & $6.18^{\mathrm{b}}$ \\
\hline 0.71 & $1046.25^{\mathrm{b}}$ & $12.68^{\mathrm{c}}$ & 82.14 & $6.48^{\mathrm{b}}$ \\
\hline SEM & 27.11 & 0.62 & 0.54 & 0.30 \\
\hline Valor $\mathrm{p}$ & 0.009 & 0.032 & 0.104 & 0.008 \\
\hline \multicolumn{5}{|c|}{$\begin{array}{l}{ }^{1} \text { Cada valor representa la media de cuatro repeticiones y cada repetición estuvo conformada por dos } \\
\text { cuyes machos } \\
{ }^{2} \text { La dieta mixta consistió en la oferta restringida de pienso (igual cantidad por repetición) + forraje ad } \\
\text { libitum } \\
\text { SEM: Error estándar de la media } \\
\text { a,b,c,d,e Las medias dentro de una fila que no comparten igual superíndice difieren significativamente } \\
\quad(p<0.05)\end{array}$} \\
\hline
\end{tabular}

Las medias de peso final, GMD, IDA e ICA se presentan en el Cuadro 4. Las mejores GMD e ICA se obtuvieron con niveles de Thr de $0.63 \%$, lo que indica que este nivel sería óptimo en alimentación mixta de cuyes en engorde, debido posiblemente al mejor aprovechamiento de nutrientes a nivel digestivo con una consecuente mayor conversión de nutrientes dietarios en peso corporal, al ser la Thr constituyente principal de la mucina y propiciadora de buena salud intestinal (Wang et al., 2010). Estos resultados concuerdan con los encontrados por Qaisrani et al. (2015) en pollos de engorde, quienes suplementaron Thr por encima del nivel recomendado por la NRC, logrando mejores tasas de crecimiento, además de mayor altura de vellosidades y mayor relación en altura de vellosidad y profundidad de criptas a nivel duodenal.

Menor incremento de peso se observó con cantidades de Thr inferiores y superiores a $0.63 \%$, pudiendo ser debido a las rela- ciones que se producen entre la Thr y los demás aminoácidos. En este sentido, Abdaljaleel et al. (2018) encontraron una proporción óptima de Thr a lisina de 0.7 en pollos de engorde que resultó en un aumento de altura de vellosidades en yeyuno y ancho de vellosidad de íleon en comparación con las otras proporciones aminoacídicas. Otras relaciones de aminoácidos que afectan el engorde de los animales ocurren entre Thr y aminoácidos azufrados (Kidd, 2000). Los niveles de lisina y metionina en el pienso en el presente estudio fueron iguales para los cinco tratamientos, pero con diferentes proporciones de Thr con relación a los demás aminoácidos. Se podría inferir que el nivel de Thr dietética de $0.63 \%$ genera una mejor relación con los demás aminoácidos del alimento que los otros niveles en estudio. No obstante, las necesidades de Thr del cuy en crecimiento podrían variar de acuerdo con la cantidad y tipo de fibra dietética. 
Cuadro 5. Rendimiento de carcasa y pesos relativos (\%) del tracto gastrointestinal (TGI), hígado, pulmones, corazón, riñones y bazo de cuyes alimentados con dieta mixta ${ }^{1}$ $80 \%$ rye grass-alfalfa y $20 \%$ pienso y sacrificados a los 72 días de edad ${ }^{2}$

\begin{tabular}{|c|c|c|c|c|c|c|c|}
\hline & \multicolumn{5}{|c|}{ Nivel de treonina en la dieta $(\%)$} & \multirow{2}{*}{ SEM } & \multirow{2}{*}{$\mathrm{p}$} \\
\hline & 0.55 & 0.59 & 0.63 & 0.67 & 0.71 & & \\
\hline $\begin{array}{l}\text { Rendimiento de } \\
\text { carcasa }\end{array}$ & $63.64^{\mathrm{b}}$ & $60.98^{d}$ & $65.56^{\mathrm{a}}$ & $62.33^{c}$ & $61.90^{c}$ & 0.79 & 0.008 \\
\hline TGI & $19.37^{\mathrm{d}}$ & $20.59^{c}$ & $17.74^{\mathrm{e}}$ & $23.34^{\mathrm{a}}$ & $21.86^{\mathrm{b}}$ & 0.98 & 0.001 \\
\hline Hígado & $3.64^{\mathrm{c}}$ & $4.41^{\mathrm{a}}$ & $3.41^{\mathrm{c}}$ & $4.04^{\mathrm{b}}$ & $3.83^{b}$ & 0.17 & 0.002 \\
\hline Pulmones & $0.63^{\mathrm{b}}$ & $0.78^{\mathrm{a}}$ & $0.75^{\mathrm{a}}$ & $0.57^{\mathrm{b}}$ & $0.73^{\mathrm{a}}$ & 0.04 & 0.006 \\
\hline Corazón & 0.30 & 0.35 & 0.32 & 0.41 & 0.36 & 0.02 & 0.064 \\
\hline Riñones & $1.19^{\mathrm{a}}$ & $1.09^{\mathrm{b}}$ & $1.07^{\mathrm{b}}$ & $1.14^{\mathrm{b}}$ & $1.20^{\mathrm{a}}$ & 0.03 & 0.009 \\
\hline Bazo & 0.19 & 0.16 & 0.16 & 0.20 & 0.18 & 0.01 & 0.517 \\
\hline \multicolumn{8}{|c|}{$\begin{array}{l}{ }^{1} \text { La dieta mixta consistió en la oferta restringida de pienso (igual cantidad por repetición) + forraje ad } \\
\text { libitum } \\
{ }^{2} \text { Cada valor representa la media de cuatro repeticiones y cada repetición estuvo conformada por un } \\
\text { cuy macho } \\
\text { SEM: Error estándar de la media }\end{array}$} \\
\hline
\end{tabular}

\section{Rendimiento de Carcasa y Peso de Ór- ganos}

Se observan diferencias entre tratamientos para la mayoría de los indicadores $(\mathrm{p}<0.05)$, excepto para los pesos relativos de corazón y de bazo (Cuadro 5).

El rendimiento de carcasa fue mayor en cuyes que consumieron dietas con $0.63 \%$ de $\operatorname{Thr}(\mathrm{p}<0.05)$, que podría ser el nivel óptimo para la mejora del RC, posiblemente debido a que la Thr interviene en la formación de tejido muscular (Jiang et al., 2020), además de estar relacionada con la expresión de los genes que regulan la deposición proteica (Jiang et al., 2019). En el presente estudio no se determinó la formación de masa muscular ni la deposición proteica, pero se encontró una mayor GMD con el nivel de $0.63 \%$ (Cuadro 4). Por otro lado, el mayor RC está relacionado con el menor peso del TGI (Cuadro 5), dado que la Thr dietética tiene un efecto directo sobre la morfología y desarrollo intestinal a través de su participación en la síntesis de mucina que recubre la capa mucosa (Wang et al., 2007).

Al sumar el peso de corazón, hígado y riñones a la carcasa como usualmente se hace, el RC de los cuyes con $0.63 \%$ de Thr sería de $70.36 \%$, el cual se encuentra dentro del rango de RC (67-71\%) que reporta Airahuacho y Vergara (2017) al alimentar cuyes con rangos de Thr entre 0.50 y $0.60 \%$ y con niveles de energía digestible de 2.7 a $3.0 \mathrm{Mcal} / \mathrm{kg}$. Si se suma el peso de corazón, hígado, riñones, pulmones y bazo el RC asciende a $71.27 \%$, mayor al $69.8 \%$ reportado por Huamaní et al. (2016) en cuyes alimentados solo con alfalfa. No obstante, el RC del tratamiento con $0.63 \%$ de Thr fue inferior al reportado en cuyes alimentados con pienso conteniendo $0.60 \%$ de Thr (Paredes et al., 2021). Por tanto, el nivel de Thr de $0.63 \%$ en dieta mixta con $80 \%$ de FV-RGA para cuyes en engorde fue el mejor. 
Por otro lado, el mayor peso relativo del TGI se obtuvo con niveles de 0.67 y $0.71 \%$ de $\mathrm{Thr}$, lo cual podría estar asociado a la función inmunológica que cumple este aminoácido (Zhang et al., 2017), por encontrarse en intestinos, tejidos y órganos linfoides, que en otras especies se conoce que a nivel intestinal se localizan las placas de Peyer, tonsilas cecales y otros tejidos donde se desarrolla respuesta inmune específica (Gómez et al., 2010; Kogut et al., 2020).

El peso relativo del hígado se vio afectado por los niveles de Thr en la dieta, lo que contrasta con el estudio de Paredes y Goicochea (2021) al alimentar cuyes en crecimiento con dietas que contenían $0.60 \%$ de Thr y diferentes proporciones de fibra detergente neutra y almidón. Del mismo modo el peso relativo de los pulmones, órganos de intercambio gaseoso y de los riñones, órganos de excreción de urea, también se vieron influenciados por los diferentes contenidos de Thr (Cuadro 5). El peso relativo del corazón no varió con los niveles de Thr dietética, tal y como reporta Airahuacho et al. (2017). El peso relativo del bazo tampoco se vio afectado por los tratamientos, debido probablemente a que los cuyes no enfrentaron mayores desafíos sanitarios (Wu et al., 2021).

\section{Conclusiones}

- Cuyes de engorde alimentados con $80 \%$ forraje verde y $20 \%$ pienso lograron mayores ganancias de peso, mejor conversión alimenticia y mayor rendimiento de carcasa con niveles de treonina dietética de $0.63 \%$.

- El tracto gastrointestinal del cuy con posibles funciones inmunológicas por contener tejido linfoide alcanzó mayor peso relativo con niveles altos de treonina $(0.67$ y $0.71 \%$ ); sin embargo, el peso del bazo no sufrió cambios posiblemente debido a que los cuyes estuvieron exentos de desafíos infecciosos.
- El peso relativo del corazón no se vio afectado por los niveles de treonina dietética; a diferencia de otros órganos de alto flujo de nutrientes y excreción de metabolitos como hígado, pulmones y riñones.

\section{Literatura Citada}

1. Abdaljaleel RA, Al-Ajeeli M, Jameel Y, Hashim MM, Bailey CA. 2018. Assessing effects of yeast cell wall supplementation on threonine requirements in broilers as measured by performance and intestinal morphology. Poultry Sci 97: 2473-2478. doi: 10.3382/ ps/pey095

2. Ahmed I, Qaisrani SN, Azam F, Pasha $T N$, Bibi F, Naveed $S$, Murtaza $S$. 2020. Interactive effects of threonine levels and protein source on growth performance and carcass traits, gut morphology, ileal digestibility of protein and amino acids, and immunity in broilers. Poultry Sci 99: 280-289. doi: 10.3382/ps/ pez488

3. Airahuacho FE, Vergara V. 2017. Evaluación de dos niveles de energía digestible en base a los estándares nutricionales del NRC (1995) en dietas de crecimiento para cuyes (Cavia porcellus L). Rev Inv Vet Perú 28: 255 264. doi: 10.15381/rivep.v28i2.13079

4. Berres J, Vieira SL, Coneglian JLB, Olmos AR, Md D, Bortolini Freitas TCK, da Silva GK. 2007. Broiler responses to graded increases in the threonine to lysine ratio. Cienc Rural 37: 510-517. doi: 10.1590/S0103-84782007000200033

5. Chauca L. 2018. Manual de crianza de cuyes. Lima: Instituto Nacional de Innovación Agraria-INIA. 80 p.

6. de Blas C, Mateos GG, GarcíaRebollar P. 2010. Tablas FEDNA de composición y valor nutritivo de alimentos para la fabricación de piensos com- 
puestos. $3^{\circ}$ ed. Madrid: Fundación Española para el Desarrollo de la Nutrición Animal. 502 p.

7. Debnath BC, Biswas P, Roy B. 2019. The effects of supplemental threonine on performance, carcass characteristics, immune response and gut health of broilers in subtropics during pre-starter and starter period. J Anim Physiol An N 103:29-40. doi: 10.1111/jpn.12991

8. Gómez G, López C, Maldonado C, Ávila E. 2010. El sistema inmune digestivo en las aves. Investig Cienc 18: 9-16.

9. Huamaní G, Zea O, Gutiérrez, G, Vilchez C. 2016. Efecto de tres sistemas de alimentación sobre el comportamiento productivo y perfil de ácidos grasos de carcasa de cuyes (Cavia porcellus). Rev Inv Vet Perú 27: 486494. doi: 10.15381/rivep.v27i3.12004

10. Ji S, Qi X, Ma S, Liu X, Min Y. 2019. Effects of dietary threonine levels on intestinal immunity and antioxidant capacity based on cecal metabolites and transcription sequencing of broiler. Animals 9: 739 . doi: 10.3390/ani9100739

11. Jiang Y, Xie M, Tang J, Zhou Z, Zhang Y, Chen G, Hou S. 2020. Effects of genetic selection and threonine on meat quality in Pekin ducks. Poultry Sci 99: 2508-2518. doi: 10.1016/j.psj.2019.10.059

12. Jiang S, El-Senousey HK, Fan Q, Lin $X$, Gou Z, Li L, Wang Y, Fouad AM, Jiang Z. 2019. Effects of dietary threonine supplementation on productivity and expression of genes related to protein deposition and amino acid transportation in breeder hens of yellow-feathered chicken and their offspring. Poultry Sci 98: 6826-6836. doi: 10.3382/ps/pez420.

13. Kheravii SK, Swick RA, Choct $M, W u$ $S B .2017$. Coarse particle inclusion and lignocellulose rich fiber addition in feed benefit performance and health of broiler chickens. Poultry Sci 96: 3272-3281. doi: 10.3382/ps/pex123
14. Kidd M. 2000. Nutritional considerations concerning threonine in broilers. World Poult Sci J 56: 139-151. doi: 10.1079/WPS20000011

15. Kogut MH, Lee A, Santin E. 2020. Microbiome and pathogen interaction with the immune system. Poultry Sci 99: 1906-1913. doi: 10.1016/j.psj.2019.12.011

16. Lievin-Le Moal V, Servin AL. 2006. The front line of enteric host defense against unwelcome intrusion of harmful microorganisms: mucins, antimicrobial peptides, and microbiota. Clin Microbiol Rev 19:315e37. doi: 10.1128/CMR.19.2.315-337.2006

17. Montagne L, Pluske JR, Hampson DJ. 2003. A review of interactions between dietary fibre and the intestinal mucosa, and their consequences on digestive health in young non-ruminant animals. Anim Feed Sci Tech 108: 95e117. doi: 10.1016/S0377-8401(03)00163-9

18. [NRC] National Research Council. 1995. Nutrient requirements of laboratory animals. $4^{\text {th }}$ rev. ed. Subcommittee on Laboratory Animal Nutrition. Washington DC. [Internet]. Available in: https://www.ncbi.nlm.nih.gov/books/ NBK231927/

19. Ortiz-Oblitas P, Florián-Alcántara A, Estela-Manrique J, Rivera-Jacinto $M$, Hobán-Vergara C, Murga-Moreno $C$. 2021. Caracterización de la crianza de cuyes en tres provincias de la Región Cajamarca, Perú. Rev Inv Vet Perú 32: e20019. doi: 10.15381/rivep.v32i2.20019

20. Paredes M, Mantilla J, Bustamante I, Mantilla JC, Cayotopa J, Hoban C, Ortiz P, Mustafa A. 2021. Efecto de cinco niveles de balance electrolítico dietario en el crecimiento, características de carcasa y metabolitos de suero sanguíneo del cuy (Cavia porcellus). Rev Inv Vet Perú 32: e20018. doi: 10.15381/ rivep.v32i2.20018

21. Paredes M, Goicochea E. 2021. Efecto de cinco dietas con diferentes proporciones de fibra detergente neutro y almidón en el rendimiento productivo, 
comportamiento ingestivo y peso de órganos digestivos del cuy (Cavia porcellus). Rev Inv Vet Perú 32: e19495. doi: 10.15381/rivep.v32i1.19495

22. Qaisrani SN, van Krimpen MM, Kwakkel RP, Verstegen MWA, Hendriks WH. 2015. Diet structure, butyric acid, and fermentable carbohydrates influence growth performance, gut morphology, and cecal fermentation characteristics in broilers. Poultry Sci 94: 2152-2164. doi: 10.3382/ps/pev003

23. Rezaeipour V, Fononi H, Irani M. 2012. Effects of dietary L-threonine and Saccharomyces cerevisiae on performance, intestinal morphology and immune response of broiler chickens. S Afr J Anim Sci 42: 266e73. doi: 10.4314/ sajas.v42i3.8

24. Ruth MR, Field CJ. 2013. The immune modifying effects of amino acids on gut-associated lymphoid tissue. J Anim Sci Biotechnol 4: 27. doi: 10.1186/2049-1891-4-27

25. Saadatmand N, Toghyani M, Gheisari A. 2019. Effects of dietary fiber and threonine on performance, intestinal morphology and immune responses in broiler chickens. Anim Nutr 5: 248-255. doi: 10.1016/j.aninu.2019.06.001

26. Serena A, Hedemann MS, Knudsen KEB. 2007. Feeding high fibre diets changes luminal environment and morphology in the intestine of sows. Livest Sci 109: 115-117. doi: 10.1016/ j.livsci.2007.01.105
27. Trevisi P, Corrent E, Mazzoni M, Messori S, Priori D, Gherpelli $Y$, Simongiovanni A, Bosi P. 2015. Effect of added dietary threonine on growth performance, health, immunity and gastrointestinal function of weaning pigs with differing genetic susceptibility to Escherichia coli infection and challen-ged with E. coli K88ac. J Anim Physiol An N 99: 511-520. doi: 10.1111/jpn.12216

28. Wang W, Zeng $X$, Mao $X, W u$ G, Qiao S. 2010. Optimal dietary true ileal digestible threonine for supporting the mucosal barrier in small intestine of weanling pigs. J Nutr 140: 981-986. doi: 10.3945/jn.109.118497.

29. Wang XU, Qiao S, Yin Y, Yue L, Wang $Z, \boldsymbol{W u} G$. 2007. A deficiency or excess of dietary threonine reduces protein synthesis in jejunum and skeletal muscle of young pigs. J Nutr 137: 1442-1446. doi: $10.1093 / \mathrm{jn} / 137.6 .1442$

30. Wu W, Qiu S, Huang H, Xu R, Bao E, LV Y. 2021. Immune-related gene expression in the kidneys and spleens of goslings infected with goose nephritic astrovirus. Poultry Sci 100: 100990. doi: 10.1016/j.psj.2021.01.013

31. Zhang Q, Chen X, Eicher SD, Ajuwon QM, Applegate TJ. 2017. Effect of threonine on secretory immune system using a chicken intestinal ex vivo model with lipopolysaccharide challenge. Poultry Sci 96: 3043-3051. doi: 10.3382/ ps/pex111 